\title{
A Temporal Galerkin Discretization of the Charge-Current Continuity Equation
}

\author{
Y. Beghein* \\ K. Cools ${ }^{\dagger}$ \\ D. De Zutter
}

\begin{abstract}
In time domain boundary integral equations, scattered fields are computed from a priori unknown electric current and charge densities. In many implementations, the charge density is eliminated from the integral equation prior to discretization, using the charge-current continuity equation. In this contribution, the charge density is explicitly discretized, and the continuity equation is weakly enforced by a space-time Galerkin procedure, leading to a simpler and more consistent implementation. The effects of this discretization scheme on the stability and the accuracy of the resulting solution method are discussed.
\end{abstract}

\section{INTRODUCTION}

Time domain boundary integral equations (TDBIEs) such as the electric, magnetic and combined field integral equations (EFIE, MFIE and CFIE, respectively) present an efficient way to model broadband and transient scattering by perfect electrical conductors. In this approach, the scattered electromagnetic fields are computed from unknowns defined only on the surfaces of the scattering objects, thus reducing the dimensionality of the problem by one. By imposing the correct boundary conditions and applying a suitable discretization to the resulting equations, a finite set of linear equations is obtained, which can be solved using the Marching-onin-Time (MoT) algorithm.

Whereas the MFIE is formulated in terms of a current density only, the EFIE and the CFIE (which is a linear combination of the EFIE and the MFIE) are defined in terms of both a current and a charge density, which together must satisfy a continuity equation. Often, this continuity equation is used to eliminate the charge density from the integral equation prior to discretization. This is revisited in Sections 2 and 3.

However, it is also possible to explicitly discretize the charge density, and to approximately enforce the charge-current continuity equation during the MoT algorithm. The advantage of this approach is that the temporal discretization of the current and

\footnotetext{
*Department of Information Technology (INTEC), Ghent University, 9000 Ghent, Belgium, e-mail: yves.beghein@ugent. be.

${ }^{\dagger}$ George Green Institute for Electromagnetics Research , Nottingham University, Nottingham, NG7 2RD, UK.

$\ddagger$ Department of Information Technology (INTEC), Ghent University, 9000 Ghent, Belgium.
}

the charge can be chosen independently. In [1], it has been shown that this additional freedom may be required to attain optimal stability. It is the aim of this contribution to further explore this approach. A higher order space-time Galerkin discretization of the continuity equation is proposed in Section 4. The effect of this discretization scheme on the stability and the accuracy of the resulting solution method is discussed using numerical examples in Section 5.

\section{THE EFIE}

Consider a perfect conductor $\Omega$ which is illuminated by an incident electromagnetic field $\left(\boldsymbol{e}^{i}(\boldsymbol{r}, t), \boldsymbol{h}^{i}(\boldsymbol{r}, t)\right)$. This field is zero in the neighborhood of $\Omega$ for $t<0$. At $t>0$, an electric current density $\boldsymbol{j}(\boldsymbol{r}, t)$ and an electric charge density $\rho(\boldsymbol{r}, t)$ are induced on the surface of the conductor, which is denoted $\Gamma$. These densities satisfy the chargecurrent continuity equation:

$$
\frac{\partial \rho}{\partial t}(\boldsymbol{r}, t)+\nabla \cdot \boldsymbol{j}(\boldsymbol{r}, t)=0, \quad \boldsymbol{r} \in \Gamma, t>0
$$

as well as the EFIE:

$$
-\eta \mathcal{T}\{\boldsymbol{j}, \rho\}(\boldsymbol{r}, t)=\hat{\boldsymbol{n}} \times \boldsymbol{e}^{i}(\boldsymbol{r}, t), \quad \boldsymbol{r} \in \Gamma, t>0,
$$

where

$$
\begin{aligned}
& \mathcal{T}\{\boldsymbol{j}, \rho\}(\boldsymbol{r}, t) \\
= & -\frac{1}{4 \pi c} \hat{\boldsymbol{n}} \times \int_{\Gamma} \frac{\partial_{t} \boldsymbol{j}\left(\boldsymbol{r}^{\prime}, t_{r}\right)}{\left|\boldsymbol{r}-\boldsymbol{r}^{\prime}\right|} d s^{\prime} \\
& -\frac{c}{4 \pi} \hat{\boldsymbol{n}} \times p . v \cdot \int_{\Gamma} \nabla \frac{\rho\left(\boldsymbol{r}^{\prime}, t_{r}\right)}{\left|\boldsymbol{r}-\boldsymbol{r}^{\prime}\right|} d s^{\prime},
\end{aligned}
$$

$t_{r}=t-\left|\boldsymbol{r}-\boldsymbol{r}^{\prime}\right| / c, c=1 / \sqrt{\epsilon \mu}$, and $\eta=\sqrt{\mu / \epsilon}$. The charge density can be eliminated from the EFIE (2) using the charge conservation law (1), resulting in:

$$
\begin{aligned}
-\eta \mathcal{T}\{\boldsymbol{j}\}(\boldsymbol{r}, t)=\hat{\boldsymbol{n}} \times \boldsymbol{e}^{i}(\boldsymbol{r}, t), \quad \boldsymbol{r} \in \Gamma, t>0, & \\
& \mathcal{T}\{\boldsymbol{j}\}(\boldsymbol{r}, t) \\
= & -\frac{1}{4 \pi c} \hat{\boldsymbol{n}} \times \int_{\Gamma} \frac{\partial_{t} \boldsymbol{j}\left(\boldsymbol{r}^{\prime}, t_{r}\right)}{\left|\boldsymbol{r}-\boldsymbol{r}^{\prime}\right|} d s^{\prime} \\
+ & \frac{c}{4 \pi} \hat{\boldsymbol{n}} \times p . v \cdot \int_{\Gamma} \nabla \frac{\int_{0}^{t_{r}} \nabla^{\prime} \cdot \boldsymbol{j}\left(\boldsymbol{r}^{\prime}, \tau\right) d \tau}{\left|\boldsymbol{r}-\boldsymbol{r}^{\prime}\right|} d s^{\prime} .
\end{aligned}
$$


For numerical solution methods, (3) has the advantage that only one unknown needs to be discretized. This, however, comes at the cost of having to discretize a temporal integral.

\section{CURRENT-ONLY DISCRETIZATION}

An approximate numerical solution to (3) can be constructed using the MoT algorithm (see e.g. [2] and references therein). First, the unknown current density $\boldsymbol{j}(\boldsymbol{r}, t)$ is expanded in a set of $N_{T}$ temporal and $N_{S}$ spatial basis functions:

$$
\boldsymbol{j}(\boldsymbol{r}, t)=\sum_{m=1}^{N_{S}} \sum_{i=1}^{N_{T}} \mathbf{j}_{m}^{(i)} \boldsymbol{f}_{m}(\boldsymbol{r}) T(t-i \Delta t),
$$

$\forall \boldsymbol{r} \in \Gamma$, where $T(t)$ is a piecewise polynomial Lagrange interpolation function [3], and $\boldsymbol{f}_{m}(\boldsymbol{r})$ are the Rao-Wilton-Glisson functions [4], which are defined on a triangulation $\mathcal{T}_{h}$ of $\Gamma$. The charge-current continuity equation then implies that

$$
\begin{aligned}
\rho(\boldsymbol{r}, t) & =-\sum_{m=1}^{N_{S}} \sum_{i=1}^{N_{T}} \mathrm{j}_{m}^{(i)} \nabla \cdot \boldsymbol{f}_{m}(\boldsymbol{r}) \tilde{T}(t-i \Delta t), \\
\tilde{T}(t) & =\int_{-\infty}^{t} T(\tau) d \tau .
\end{aligned}
$$

By evaluating (3) at $t=i \Delta t$ (temporal collocation method) and applying a spatial Galerkin procedure, one obtains a system of linear equations of the following form:

$$
\mathbf{Z}_{0} \mathrm{j}^{(i)}=\mathrm{v}^{i}-\sum_{l=1}^{i} \mathbf{Z}_{l \mathbf{j}^{(i-l)}}
$$

The MoT algorithm consists of successively solving this equation for $i=1,2,3, \ldots, N_{T}$.

Note that the support of $\tilde{T}(t)$ is not bounded. A naive discretization therefore leads to a system of equations that is not sparse.

Additionally, the stability of this collocation-intime scheme is very sensitive to the discretization technique that is being used: only first order (piecewise linear) interpolation functions yield stable MoT schemes. It is therefore not possible to increase the accuracy of the simulations by using higher order Lagrange interpolators.

Alternatively, the EFIE (3) can be temporally discretized using a Galerkin method. It was shown in [5] that this method can be extended to higher order without compromising the stability of the MoT algorithm. Due to the finite support of the chosen temporal testing functions, the charge integral is limited to a single time step, leading to a sparse system of equations. This, however, introduces an unphysical null space, leading to a spurious static component in the solution.

\section{CHARGE DISCRETIZATION}

\subsection{First Order Galerkin Method}

In contrast to the implicit charge discretization (5), the following expansion is now proposed:

$$
\rho(\boldsymbol{r}, t)=\sum_{\alpha \in \mathcal{T}_{h}} \sum_{i=1}^{N_{T}} \mathbf{q}_{\alpha}^{(i)} \mathbb{1}_{\alpha}(\boldsymbol{r}) T(t-i \Delta t),
$$

where $\mathbb{1}_{\alpha}(\boldsymbol{r})$ is 1 on triangle $\alpha$, and 0 on the others, and $T(t)$ is the hat function (first order interpolation function). This expansion has the advantage that due to the finite support of $T(t)$, only a small number of terms contribute to the charge at any given time $t$.

The charge-current continuity equation (1) cannot be imposed at all times, simply because the integral of the temporal basis function cannot be represented as a linear combination of temporal basis functions of the same order. It can, however, be enforced in the weak sense by means of a space-time Galerkin procedure: it is multiplied by $\mathbb{1}_{\beta}(\boldsymbol{r}) u(t-k \Delta t)$, and integrated over both space and time, with

$$
u(t)=\left\{\begin{array}{ll}
1, & t<0 \\
0, & t \geq 0
\end{array} .\right.
$$

This results in the following integration rule:

$$
\begin{aligned}
\mathbf{q}_{\alpha}^{(k)} & =\mathbf{q}_{\alpha}^{(k-1)}-\sum_{m} \mathbf{D}_{\alpha m} \frac{\Delta t}{2}\left(\mathrm{j}_{m}^{(k-1)}+\mathbf{j}_{m}^{(k)}\right)(9) \\
\mathbf{D}_{\alpha m} & =\int_{\Gamma} \mathbb{1}_{\alpha}(\boldsymbol{r}) \nabla \cdot \boldsymbol{f}_{m}(\boldsymbol{r}) d s / \int_{\Gamma} \mathbb{1}_{\alpha}(\boldsymbol{r}) d s
\end{aligned}
$$

Thus, the Galerkin method leads to the trapezoid rule for discretizing the temporal integral. Next, a space-time Galerkin procedure is applied to the EFIE (2): its temporal derivative is multiplied by $\hat{\boldsymbol{n}} \times \boldsymbol{f}_{n}(\boldsymbol{r}) u(t-i \Delta t)$, and integrated over both space and time, leading to an equation of the form

$$
\mathbf{Z}_{0}^{j} \mathrm{j}^{(i)}+\mathbf{Z}_{0}^{\rho} \mathbf{q}^{(i)}=\mathbf{v}^{(i)}-\sum_{l=1}^{i} \mathbf{Z}_{l}^{j} j^{(i-l)}-\sum_{l=1}^{i} \mathbf{Z}_{l}^{\rho} \mathbf{q}^{(i-l)}
$$

which can be solved simultaneously with (9). Numerical experiments show that the resulting MoT scheme is stable for a wide range of geometries. The fact that the charge-current continuity equation is only approximately fulfilled, does not lead to instability.

\subsection{Higher Order Interpolation Functions}

Using higher order interpolation functions for the current density leads to different integration rules. 
For example, if the current is expanded in second order Lagrange interpolators, (9) becomes:

$\mathbf{q}_{\alpha}^{(k)}=\mathbf{q}_{\alpha}^{(k-1)}+\sum_{m} \mathbf{D}_{\alpha m} \frac{\Delta t}{12}\left(\mathrm{j}_{m}^{(k-2)}-8 \mathrm{j}_{m}^{(k-1)}-5 \mathrm{j}_{m}^{(k)}\right)$.

Unfortunately, as was the case in the traditional discretization, this leads to unstable MoT schemes, regardless of the interpolation order used for the charge.

\subsection{Higher Order Galerkin Method}

The first order Galerkin method can be extended to higher order. As in [5], the unknowns are now expanded in two sets of temporal basis functions:

$\boldsymbol{j}(\boldsymbol{r}, t)=\sum_{m=1}^{N_{S}} \sum_{\beta=1}^{2} \sum_{i=1}^{N_{T}} \mathrm{j}_{m}^{(i, \beta)} \boldsymbol{f}_{m}(\boldsymbol{r}) T^{(\alpha)}(t-i \Delta t)$, $\rho(\boldsymbol{r}, t)=\sum_{\alpha \in \mathcal{T}_{h}} \sum_{\beta=1}^{2} \sum_{i=1}^{N_{T}} \mathbf{q}_{m}^{(i, \beta)} \mathbb{1}_{\alpha}(\boldsymbol{r}) T^{(\alpha)}(t-i \Delta t)$,

with $T^{(1)}(t)$ and $T^{(2)}(t)$ defined as in [5]. The charge-current continuity equation and the timedifferentiated EFIE are both temporally tested with $v^{(1)}(t)$ and $v^{(2)}(t)$ :

$$
\begin{aligned}
& v^{(1)}(t)= \begin{cases}1 & t<0 \\
0 & \text { otherwise }\end{cases} \\
& v^{(2)}(t)= \begin{cases}1 & t<-\Delta t \\
-\frac{t}{\Delta t} & -\Delta t<t<0 \\
0 & \text { otherwise }\end{cases}
\end{aligned}
$$

The continuity equation leads to the following integration rule:

$$
\begin{aligned}
\mathbf{q}_{\alpha}^{(k, 1)} & =\mathbf{q}_{\alpha}^{(k-1,2)} \\
& -\frac{\Delta t}{24} \sum_{m} \mathbf{D}_{\alpha m}\left(5 \mathrm{j}_{m}^{(k-1,2)}-8 \mathrm{j}_{m}^{(k, 1)}+\mathrm{j}_{m}^{(k, 2)}\right), \\
\mathbf{q}_{\alpha}^{(k, 2)} & =\mathbf{q}_{\alpha}^{(k-1,2)} \\
& -\frac{\Delta t}{6} \sum_{m} \mathbf{D}_{\alpha m}\left(\mathrm{j}_{m}^{(k-1,2)}+4 \mathrm{j}_{m}^{(k, 1)}+\mathrm{j}_{m}^{(k, 2)}\right) .
\end{aligned}
$$

It can again be solved simultaneously with the discretized EFIE. As was the case for the first order scheme, numerical experiments show that the approximation of the continuity equation does not lead to instability.

\section{NUMERICAL RESULTS}

In order to compare the accuracy of the different schemes, consider a perfectly conducting sphere of radius 1 meter, which is illuminated by a Gaussian plane wave. The exact solution (given by the

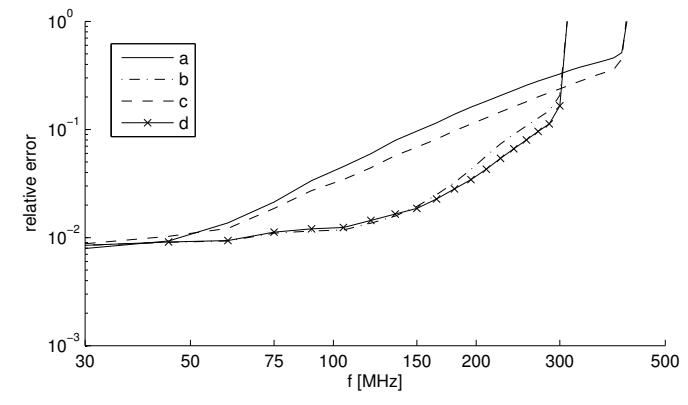

Figure 1: Accuracy of the CFIE with $(\mathrm{a}, \mathrm{b})$ and without (c, d) explicit charge discretization.

Mie series) is compared to the results obtained using the time domain CFIE, temporally discretized using the following schemes:

a. first order Galerkin discretization of both current and charge (Section 4.1, $\Delta t=0.83 n s$ ),

b. second order Galerkin discretization of both current and charge (Section 4.3, $\Delta t=1.67 n s$ ),

c. first order Galerkin discretization of current only ([5], $\Delta t=0.83 n s)$,

d. second order Galerkin discretization of current only ([5], $\Delta t=1.67 n s)$.

In each of the simulations, the sphere was approximated by a triangle mesh consisting of 666 faces, on which 999 RWG functions were defined. The relative $L^{2}$-error on the currents with respect to the Mie series solution is shown inf Figure 1. The CFIE is used instead of the EFIE to avoid spurious internal resonances $[6,7]$.

Despite the fact that the continuity equation is not imposed exactly, the accuracy of the new method is comparable to that of the solution schemes in which the continuity equation is fulfilled exactly.

\section{CONCLUSIONS}

A novel approach to the discretization of time domain boundary integral equations was introduced. In this approach, the charge-current continuity equation is not enforced exactly, but only approximately by a temporal Galerkin method. The resulting numerical integration rule is easier to implement and more efficient than the exact evaluation of the charge integral. It has been shown that the resulting scheme is stable and that the accuracy of the solution is comparable to that of solutions to the classic schemes 


\section{Acknowledgments}

The work of Y. Beghein is supported by a doctoral grant from the Agency for Innovation by Science and Technology in Flanders (IWT).

\section{References}

[1] I. Terrasse, Résolution mathématique et numérique des équations de Maxwell instationnaires par une méthode de potentiels retardés. PhD thesis, Ecole Polytechnique, 1993.

[2] W. Chew, E. Michielssen, J. M. Song, and J. M. Jin, eds., Fast and Efficient Algorithms in Computational Electromagnetics. Norwood, MA, USA: Artech House, Inc., 2001.

[3] B. Shanker, A. Ergin, K. Aygun, and E. Michielssen, "Analysis of transient electromagnetic scattering phenomena using a twolevel plane wave time-domain algorithm," IEEE Trans. Antennas and Propagation, vol. 48, pp. $510-523$, apr 2000 .

[4] S. Rao, D. Wilton, and A. Glisson, "Electromagnetic scattering by surfaces of arbitrary shape," IEEE Trans. Antennas Propag.,, vol. 30, pp. 409 - 418, may 1982.

[5] Y. Beghein, K. Cools, H. Bağc1, and D. De Zutter, "A space-time mixed Galerkin marchingon-in-time scheme for the time domain combined field integral equation," IEEE Transactions on Antennas and Propagation, 2013.

[6] W. C. Chew and J. M. Song, "Gedanken experiments to understand the internal resonance problems of electromagnetic scattering.," Electromagnetics, vol. 27, no. 8, pp. 457 - 471, 2007.

[7] J. R. Mautz and R. F. Harrington, "H-field, E-field, and combined-field solutions for conducting bodies of revolution," Archiv Elektronik und Uebertragungstechnik, vol. 32, pp. 157-164, Apr. 1978. 